\title{
REVIEW
}

\section{Intraepidermal Nerve Fiber Density: Diagnostic and Therapeutic Relevance in the Management of Chronic Pruritus: a Review}

\author{
Manuel P. Pereira · Sebastian Mühl · Esther M. Pogatzki-Zahn • \\ Konstantin Agelopoulos · Sonja Ständer
}

Received: August 11, 2016 / Published online: October 11, 2016

(C) The Author(s) 2016. This article is published with open access at Springerlink.com

\begin{abstract}
In recent years, measurement of the intraepidermal nerve fiber (IENF) density has gained relevance in the diagnostics of chronic pruritus. This method allows the objectification and quantification of a small-fiber neuropathy, which may manifest clinically with pruritus, pain or dysesthetic sensory symptoms, such as burning, stinging and tingling sensations or numbness. Upon suspicion of a small-fiber neuropathy as a cause for chronic pruritus, targeted diagnostic procedures are essential for the early detection of the neuroanatomical changes. After a punch biopsy of the lower leg,
\end{abstract}

Enhanced content To view enhanced content for this article go to http://www.medengine.com/Redeem/ 21E6F0603B053B37.

M. P. Pereira $(\bowtie) \cdot$ S. Mühl · K. Agelopoulos .

S. Ständer

Department of Dermatology and Center for Chronic Pruritus, University Hospital Münster,

Von-Esmarch-Str. 58, 48149 Münster, Germany e-mail:

ManuelPedro.FernandesLoboPereira@ukmuenster.de

E. M. Pogatzki-Zahn

Department of Anaesthesiology, Intensive Care and

Pain Medicine, University Hospital Münster,

Albert-Schweitzer-Campus 1, A1, 48149 Münster,

Germany the obtained tissue undergoes an immunofluorescence staining process with a primary antibody against the protein gene product 9.5. The IENFs can thus be detected and are quantified according to pre-determined guidelines based on an international consensus. In addition to morphological changes, functional impairment of small-fibers can be assessed using quantitative sensory testing by assessing detection and pain thresholds of various thermal and mechanic modalities. This method, however, is time-consuming and requires a specialized investigator, and thus it is not routinely used in the diagnostic investigation of chronic pruritus. Diagnosing a small-fiber neuropathy underlying chronic pruritus has therapeutic relevance. If possible, the underlying cause of the neuropathy should be treated. Alternatively, symptomatic therapy options include topical (capsaicin) and systemic (anticonvulsants and/or antidepressants) agents. Chronification processes may lead to refractory pruritus, and thus treatment should be initiated as soon as possible. The aim of this review is to present and discuss the measurement of the IENF density as a diagnostic tool and its role in the 
management of patients with chronic pruritus. A brief case report is presented to better illustrate the role of this diagnostic method in the clinical setting.

Keywords: Anticonvulsants; Capsaicin; Case report; Chronic pruritus; Corneal confocal microscopy; Intraepidermal nerve fiber density; Neuropathic pruritus; Quantitative sensory testing; Review; Small fiber neuropathy

\section{INTRODUCTION}

It is estimated that neuropathic pruritus, i.e. pruritus caused by disorders of the somatosensory system, accounts for approximately $8 \%$ of cases of chronic pruritus, while other possible causes include dermatological, systemic, psychogenic or multifactorial conditions, or may arise from unknown causes [1]. The assessment of the intraepidermal nerve fiber density (IENFD) allows the morphological quantification of the unmyelinated C-fibers crossing the basement membrane of the epidermis. This diagnostic tool has gained interest in recent years in the diagnostics of neuropathic pruritus, since, next to central processes, a dysfunction of the small unmyelinated C-fibers plays a central role in neuropathic pruritus [2]. In addition to pure itch, damage to these nerve fibers can lead to positive and negative neurological symptoms that are generally more pronounced distally. Interestingly, different cutaneous C fiber subgroups and mediators are responsible for various subjective qualities of chronic pruritus [3]. Mechano-insensitive $\mathrm{C}$ fibers (CMi fibers) utilize $\mathrm{H}_{1}$ receptors to mediate histamine-induced pruritus through the ion channel TRPV1 (transient receptor potential channel V1) and phospholipase- $\beta 3$ generating a more pure itching, while mechano- and heat-sensitive $(\mathrm{CMH}) \mathrm{C}$ fibers mediate pruritus, burning and thermal pain through the activation of TRPV1 receptors [4]. The aim of this review is to present the methodological aspects of the quantification of IENFD, as well as its diagnostic and therapeutic relevance for the clinical practice. Additionally, a brief case report is presented to demonstrate the role of this diagnostic tool in the management of patients with chronic neuropathic pruritus.

\section{When to Assess the Intraepidermal Nerve Fiber Density?}

A reduced IENFD can be found in small-fiber neuropathy of different origins, with pruritus as the leading symptom. This reinforces the pathophysiological concept that the genesis of pruritus originates from unmyelinated nerve endings in the epidermis and at the dermoepidermal junction [2].

A detailed medical history is essential for the diagnosis and further differentiation of neuropathic pruritus. In neuropathic pruritus, pathological processes causing itch may arise from different levels of the somatosensory system from the periphery to conditions of the central nervous system [5], leading to a multitude of possible clinical presentations. Depending on the pathophysiological mechanisms, patients may present a localized (e.g., in compression syndromes such as brachioradial pruritus and burning mouth syndrome) or generalized (e.g., in a generalized small-fiber neuropathy) pruritic condition [6, 7]. Typical for neuropathic pruritus is the report of sensory symptoms such as burning, itching, stinging and even pain by the affected patients. Additionally, the application of ice packs or cold water often relieves the itching, while warmth may worsen the symptoms [2]. 
Comorbidities associated with small-fiber neuropathy should be thoroughly assessed, especially its chronological association with the onset of pruritus. Metabolic diseases often induce small fiber neuropathy, for which diabetes mellitus represents the most common cause. Pruritus arising from diabetes mellitus typically begins at the feet and lower legs or at the torso [8]. Adjusting the postprandial glucose levels generally provide relief from the pruritus [9]. Other commonly associated diseases include neurological syndromes. Compression syndromes, such as brachioradial pruritus (compression of the root ganglia or spinal nerve C3-C6) and notalgia paresthetica (compression of the dorsal rami Th2-Th6) are associated with a reduced IENFD and thus to localized pruritus in the affected dermatomes. Other localized neuropathic syndromes leading to small-fiber neuropathy and pruritus include postherpetic neuralgia, vulvodynia or burning mouth syndrome [6]. Moreover, skin diseases have been linked to an impairment of small-fibers. In sensitive skin, a condition in which paraesthesias occur in apparently normal skin mostly at the face, a reduced IENFD could be demonstrated [10] and thus a neuropathic involvement is speculated [11]. Prurigo nodularis, in which itching hyperkeratotic nodules develop due to chronic scratching, is associated with a reduced IENFD regardless of the underlying pruritic disease [12]. Interestingly, with improvement of the prurigo condition, the IENFD may normalize [13].

Many other diseases are associated with small-fiber neuropathy without, however, necessarily causing itch. These include metabolic (e.g., vitamin B12 deficiency), neurological (e.g., inflammatory demyelinating diseases) immunological (e.g., rheumatoid arthritis, lupus erythematodes, M. Sjögren), infectious (e.g., hepatitis C, HIV), paraneoplastic and drug-induced (e.g., antibiotics, antiretroviral drugs, alcohol) conditions $[2,14]$.

When the detailed clinical history provides hints of a small-fiber neuropathy as the cause for pruritus, the measurement of IENFD is the gold standard for the objectification and quantification of the neuropathy [15].

\section{How to Assess the Intraepidermal Nerve Fiber Density?}

\section{Punch Biopsy}

The measurement of the IENFD is a well-established standardized laboratory method to quantify small-fiber neuropathy. European guidelines addressing this issue provide detailed recommendations for the biopsy site, methodology of the employed immunohistochemical staining and quantification of the IENFD [16, 17]. The diagnostics are carried out with a 3-mm-diameter punch biopsy. The anatomical localization of the biopsy site is of paramount importance for the measurement of the IENFD [15]. Age and gender-adapted standard value tables with cutoff values for the diagnosis of small fiber neuropathy are, until now, only available for the lower leg $[15,18]$. As a result of this, the punch biopsy to assess IENFD is usually performed at the standardized location in the distal lower leg $(10 \mathrm{~cm}$ above the lateral malleolus) [17]. For skin biopsies from other parts of the body, the guidelines recommend conducting a biopsy of a similar, unaffected site in order to compare the results of the affected to the unaffected area [17]. A biopsy can be taken from either the right or the left distal lower leg, as this does not influence the IENFD [19]. An additional biopsy from the lateral proximal thigh $(20 \mathrm{~cm}$ below the anterior superior iliac 
spine) can provide information on the proximal distribution of the neuropathy [17].

\section{Laboratory Work-Up}

The skin probe obtained from the punch biopsy is immediately fixed in paraformaldehyde and is transported to the laboratory. Here, immunohistochemical staining for discerning intraepidermal nerve fibers is carried out. An immunohistochemical or immunofluorescence staining with a primary antibody against the axonal marker protein gene product 9.5 (PGP 9.5 ) is the most commonly used method $[16,17]$, for which standard values have been established for the distal lower leg [15].

\section{Counting Method}

Precise instructions on how to quantify the IENFD have been established in an international consensus. Per tissue samples, three sections in high magnification $(\times 200-400)$ are considered. Only single intraepidermal nerve fibers that cross the dermoepidermal junction are taken into account while secondary branching or fragments are not counted. The IENFD is then determined by dividing the number of intraepidermal nerve fibers crossing the dermoepidermal junction to the length of the dermoepidermal junction (Fig. 1) [16, 17].

\section{Normative Values}

Age- and gender-specific variation exists in the IENFD. While the IENFD seems to decline with age, women show higher IENFD compared to men [17]. These aspects have been taken into consideration in the establishment of standard normative values. Cutoff values are thus stratified according to age per decade and gender, displaying $5 \%$ percentiles of the IENFD for each group (Table 1) [15, 18].
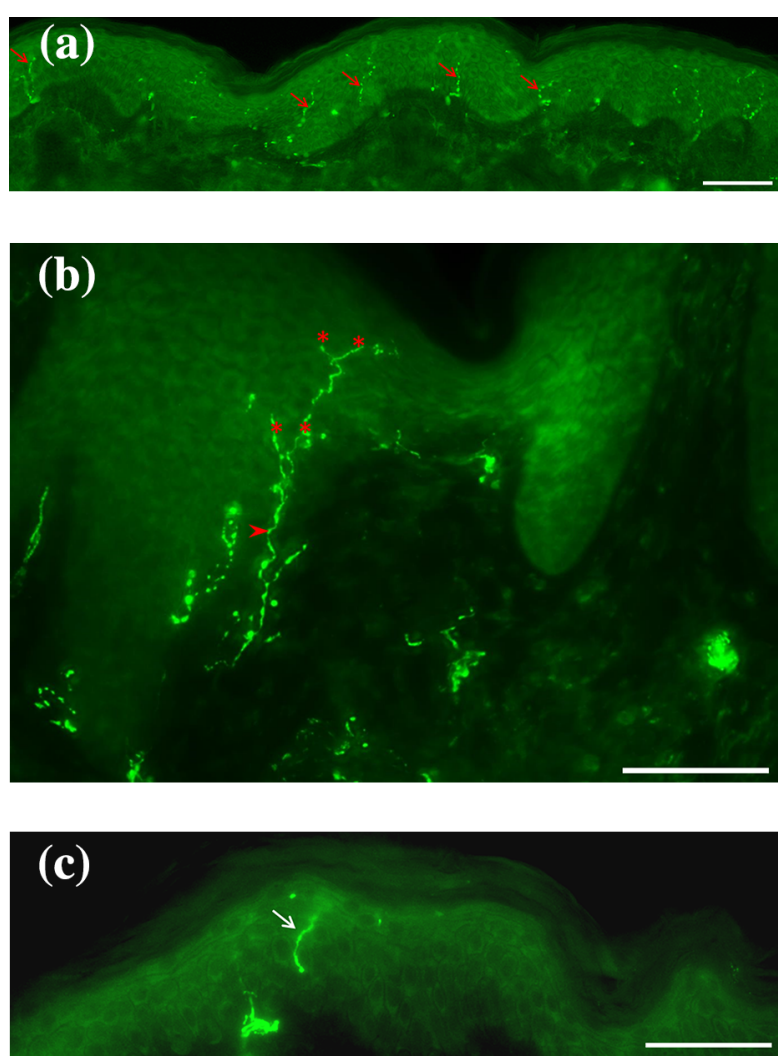

Fig. 1 PGP 9.5 intraepidermal nerve fibers. a Example of PGP 9.5 intraepidermal nerve fibers (red arrows). b Only single intraepidermal nerve fibers crossing the dermoepidermal junction (arrowhead) are taken into account. Secondary branching (asterisks) or $\mathbf{c}$ fragments (white arrow) are not counted. Magnification: $\times 200$, scale bar $100 \mu \mathrm{m}$ in $(\mathbf{a}) ; \times 400$, scale bar $100 \mu \mathrm{m}$ in $(\mathbf{b}, \mathbf{c})$. PGP 9.5 protein gene product 9.5

Table 1 Normative values for the intraepidermal nerve fiber density assessed at the distal lower leg $(10 \mathrm{~cm}$ above the lateral malleolus) according to [14]

\begin{tabular}{lll}
\hline Age group (years) & Female $(\mathbf{F} / \mathbf{m m})$ & Male $(\mathbf{F} / \mathbf{m m})$ \\
\hline $18-39$ & $>11.65$ & $>10.65$ \\
$40-59$ & $>10.55$ & $>9.55$ \\
$60-69$ & $>9.8$ & $>8.8$ \\
$>70$ & $>9.2$ & $>8.2$ \\
\hline
\end{tabular}

$F$ fibers

The localization of the obtained tissue also influences the intraepidermal nerve fiber density. Currently normative values are only 
available for the distal lower leg. However, a study is underway characterizing the density of intraepidermal nerve fibers in various body locations, both in healthy control subjects and in patients with atopic eczema and neuropathic pruritus (study ID: DRKS00010520).

Another aspect to consider is the laboratory method used to identify the intraepidermal nerve fibers, since the IENFD can vary depending on the utilized staining method. The immunofluorescence method tends to display higher IENFD values compared to the bright field staining method [20]. Normative value table have been developed for both methods $[15,18]$.

\section{Altered Neuroanatomy in Pruritic Diseases}

Neuroanatomical changes have been reported for different pruritic diseases, including atopic dermatitis or prurigo nodularis, in which dermal hyperinnervation occurs. These alterations in neuroanatomy are associated with functional impairment and with itch induction. An imbalance of nerve elongation factors (e.g., nerve growth factor) and nerve repulsion factors (e.g., semaphorin $3 \mathrm{~A}$ ) is thought to lead to the development of neuroanatomical changes [21]. Although hyperinnervation of dermal nerves has been found in chronic pruritus diseases and prurigo nodularis, the number of intraepidermal nerve fibers crossing the basal membrane are reduced compared to healthy individuals [22]. Here, scratching behavior leading to a rarefication of epidermal nerves may play a role [12]. Importantly, different methods analyzing changes of intraepidermal nerve fibers have been developed. The method quantifying the intraepidermal nerve fiber density described above differs from other methods, in which enhanced intraepidermal sprouting is assessed.

\section{Other Diagnostic Tools}

Other methods exist to quantify sensory dysfunction of cutaneous nerves; however, they find only limited use in the assessment of chronic pruritus. Quantitative sensory testing (QST) allows the functional examination of the peripheral nerve fibers and is thus complementary to morphological methods [23]. This non-invasive method consists of a battery of tests assessing the subjective somatosensory response to graded stimuli of different modalities [24-26], allowing the examination of functional impairment of peripheral and central nerve tracts, as well as central modulation mechanisms, especially central sensitization [23]. For the study of pruritic conditions, dysfunction of unmyelinated C-fibers and thinly myelinated A $\delta$-fibers are of special interest. C-fibers are activated by non-noxious heat stimulation as well as by noxious cold and heat, while A $\delta$-fibers are activated by pinprick stimulation, noxious heat and non-noxious cold stimulation. At peripheral level, activation of unencapsulated receptors occurs and signals are centrally transmitted by the anterolateral spinothalamic tract to higher centers $[25,26]$. The German Research Network on Neuropathic Pain has established QST as a viable and standardized protocol [23] that is now well established for adults and with modifications for children [27]. Recently, QST was officially recommended by the Neuropathic Pain Special Interest Group of the International Association for the Study of Pain both in research as well as in the clinical evaluation of peripheral and central neuropathies [24]. Notably, QST should not be used as a stand-alone test for the diagnosis of neuropathic pain.

Other functional methods assessing the function of C- and A $\delta$-fibers include 
somatosensory evoked potentials, in which nerve transmission dysfunctions from peripheral to central levels of the somatosensory system can be detected [28], as well as other diagnostic tools used mainly for research purposes, such as laser-evoked potentials, microneurography and the neurometer. On the other hand, nerve conduction studies, in which the function of large myelinated $A \alpha$ and $A \beta$ fibers is assessed [29], are routinely used in neurology departments. Although this method is not usually used in the assessment of chronic pruritus, nerve conduction studies may be useful in the characterization of compression syndromes, such as brachioradial pruritus [30] or lumbosacral radiculopathy causing anogenital pruritus [31]. This method is, however, unable to detect a small-fiber neuropathy, which is often associated with compression syndromes inducing pruritus but can be nicely combined with QST to give a comprehensive functional overview.

In addition to the determination of IENFD, another morphological method has been developed in recent years. Corneal confocal microscopy enables the study of morphological changes in $\mathrm{A} \delta$ and $\mathrm{C}$ fibers belonging to the subbasal nerve plexus between the basal epithelium and Bowman's membrane [32-34]. Using a microscope connected to a retina tomograph, the density of the nerve fibers (nerve fibers $/ \mathrm{mm}^{2}$ ) $[35,36]$ as well as the length and branching are measured in order to assess a possible small-fiber neuropathy. Although this method is expensive and only available in few centers, it has shown to be useful in the early diagnosis of diabetic neuropathy [37].

\section{Therapeutic Relevance of the Cutaneous} IENFD

An IENFD below the 5\% percentile of the corresponding gender and age argues for a small fiber neuropathy. However, this finding alone is not sufficient for the diagnosis of a small-fiber neuropathy. A suitable clinical history is essential for the diagnosis of a small-fiber neuropathy underlying chronic pruritus. Importantly, chronic scratching behavior, for instance, can also cause a reduced IENFD [12] and should be taken into consideration when interpreting the results of this investigation.

An early start of the treatment is essential to its effectiveness. It is recommended that the attending physician initiates a directed treatment against a suspected small-fiber neuropathy, even before confirmation with objective diagnostic methods such as the measurement of the IENFD. If the cause of the neuropathy is known, treatment of the underlying condition is the most effective measure. It is important to emphasize that determination of the etiology of small fiber neuropathy cannot be drawn based on the morphological assessment of the IEFND [17]. If it is not possible to identify and treat the underlying cause, the European as well as the German guidelines recommend a symptomatic approach. The application of capsaicin cream in rising concentrations, or, alternatively, a capsaicin $8 \%$ patch, is the first-line treatment for localized pruritus [38, 39], as was the case in the brief case report presented in Fig. 2. Capsaicin acts by destroying superficial sensory nerves, depleting them from neuropeptides and finds application in various conditions, in which localized neurogenic 


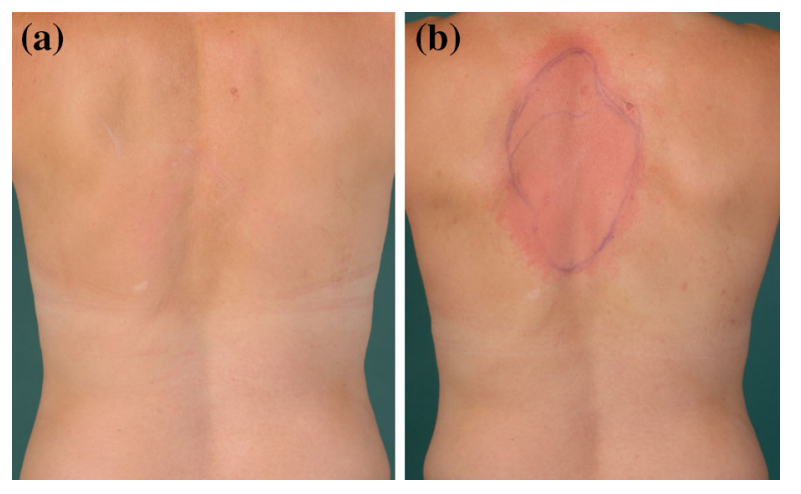

Fig. 2 Brief case report: application of an $8 \%$ capsaicin patch. To better illustrate the role of the measurement of the intraepidermal nerve fiber density in the management of neuropathic pruritus, we present a brief case report. Informed consent was obtained from the patient for being included in the study. Medical history: A 45-year-old female presented with localized pruritus at the upper back between the scapulae. In addition to itch, the patient reported a tingling and stinging sensation. These sensory symptoms were of moderate intensity $(5-6 / 10$ in the visual analogue scale), but could become very intense during attacks (up to $9 / 10$ in the visual analogue scale). Skin status: Upon examination of the skin, discrete erythematous lesions, likely due to scrubbing and scratching, could be observed in an otherwise normal skin. The dermographism was white. Intraepidermal nerve fiber density: Skin biopsies were taken at the back both in a lesional and a non-lesional area: lesional skin probe: 2.41 fibers $/ \mathrm{mm}$ (strongly reduced IENFD) and non-lesional skin probe: 14.30 fibers $/ \mathrm{mm}$ (normal IENFD). Diagnose: Notalgia paraesthetica. Previous therapies: A treatment with antihistamines did not alleviate the symptoms. The patient did not tolerate pregabalin due to nausea and dizziness. Gabapentin (up to $900 \mathrm{mg} /$ day) as well as paroxetine $(20 \mathrm{mg} /$ day $)$ showed no effect. Proposed treatment: Due to the localized sensory symptoms, an $8 \%$ capsaicin patch was applied in the affected area. The skin condition of the affected area is shown before (a) and after (b) application of the patch. A long-lasting itch relief is expected with this treatment. However, the application of the capsaicin patch may be repeated every 3 months or at longer intervals, if needed. Additionally, a prescription for a capsaicin cream in rising concentrations $(0.025,0.05$ and $0.075 \%$ ) was given to the patient to be used in case of itch recurrence pruritus or pain is present [40]. This treatment may be repeated every 3 months, if necessary. If the topical treatment does not lead to an improvement of the symptom or in case of generalized pruritus, the systemic administration of anticonvulsants (e.g., gabapentin and pregabalin) should be considered. These may be combined with antidepressants such as paroxetine, mirtazapine or amitriptyline, if additional therapeutic effect is needed [38, 39].

\section{CONCLUSION}

Chronic pruritus may arise from a dysfunction of small nerve fibers. In combination with a detailed medical history, the determination of the intraepidermal nerve fiber density in punch biopsies is a simple well-established method to detect a small-fiber neuropathy. An early diagnosis of a neuropathy as the underlying cause of pruritus is essential for the treatment success. Current guidelines recommend the treatment of the underlying cause or, if not possible, the application of topical capsaicin or the systemic use of anticonvulsants or antidepressants.

\section{ACKNOWLEDGMENTS}

We thank E.R. Burnett for proofreading and editing this manuscript. This work was supported by a grant from the Interdisciplinary Center for Clinical Research (IZKF; CTRP 07). All named authors meet the International Committee of Medical Journal Editors (ICMJE) criteria for authorship for this manuscript, take responsibility for the integrity of the work as a whole, and have given final approval to the version to be published. 
Disclosures. M.P. Pereira, S. Mühl, E.M. Pogatzki-Zahn, K. Agelopoulos and S. Ständer declare that they have no conflicts of interest.

Compliance with Ethics Guidelines. Informed consent was obtained from the patient for being included in the study.

Open Access. This article is distributed under the terms of the Creative Commons Attribution-NonCommercial 4.0 International License (http://creativecommons.org/licenses/ by-nc/4.0/), which permits any noncommercial use, distribution, and reproduction in any medium, provided you give appropriate credit to the original author(s) and the source, provide a link to the Creative Commons license, and indicate if changes were made.

\section{REFERENCES}

1. Stander S, Weisshaar E, Mettang T, et al. Clinical classification of itch: a position paper of the International Forum for the Study of Itch. Acta Derm Venereol. 2007;87(4):291-4.

2. Brenaut E, Marcorelles P, Genestet S, Menard D, Misery L. Pruritus: an underrecognized symptom of small-fiber neuropathies. J Am Acad Dermatol. 2015;72(2):328-32.

3. Baraniuk JN. Rise of the sensors: nociception and pruritus. Curr Allergy Asthma Rep. 2012;12(2):104-14.

4. Ringkamp M, Meyer R. Pruriceptors. In: Carstens E, Akiyama $\mathrm{T}$, editors. Itch: mechanisms and treatment. Boca Raton: CRC Press/Taylor \& Francis; 2014.

5. Stander S, Schmelz M. Chronic itch and painsimilarities and differences. Eur J Pain. 2006;10(5):473-8.

6. Braud A, Toure B, Agbo-Godeau S, Descroix V, Boucher Y. Characteristics of pain assessed with visual analog scale and questionnaire in burning mouth syndrome patients: a pilot study. J Orofac Pain. 2013;27(3):235-42.
7. Stander S. Chronic pruritus: principals of diagnostics and therapy. Hautarzt. 2007;58(7):627-36 (quiz 37).

8. Yamaoka H, Sasaki H, Yamasaki H, et al. Truncal pruritus of unknown origin may be a symptom of diabetic polyneuropathy. Diabetes Care. 2010;33(1):150-5.

9. Ko MJ, Chiu HC, Jee $\mathrm{SH}, \mathrm{Hu}$ FC, Tseng $\mathrm{CH}$. Postprandial blood glucose is associated with generalized pruritus in patients with type 2 diabetes. Eur J Dermatol. 2013;23(5):688-93.

10. Buhe V, Vie K, Guere C, et al. Pathophysiological study of sensitive skin. Acta Derm Venereol. 2016;96(3):314-8.

11. Misery L, Loser K, Stander S. Sensitive skin. J Eur Acad Dermatol Venereol. 2016;30(Suppl 1):2-8.

12. Schuhknecht B, Marziniak M, Wissel A, et al. Reduced intraepidermal nerve fibre density in lesional and nonlesional prurigo nodularis skin as a potential sign of subclinical cutaneous neuropathy. Br J Dermatol. 2011;165(1):85-91.

13. Bobko S, Zeidler C, Osada $\mathrm{N}$, et al. Intraepidermal nerve fibre density is decreased in lesional and inter-lesional prurigo nodularis and reconstitutes on healing of lesions. Acta Derm Venereol. 2016;96(3):404-6.

14. Lauria G, Merkies IS, Faber CG. Small fibre neuropathy. Curr Opin Neurol. 2012;25(5):542-9.

15. Provitera V, Gibbons $\mathrm{CH}$, Wendelschafer-Crabb G, et al. A multi-center, multinational age- and gender-adjusted normative dataset for immunofluorescent intraepidermal nerve fiber density at the distal leg. Eur J Neurol. 2016;23(2):333-8.

16. Lauria G, Cornblath DR, Johansson O, et al. EFNS guidelines on the use of skin biopsy in the diagnosis of peripheral neuropathy. Eur J Neurol. 2005; 12(10):747-58.

17. Lauria G, Hsieh ST, Johansson O, et al. European Federation of Neurological Societies/Peripheral Nerve Society Guideline on the use of skin biopsy in the diagnosis of small fiber neuropathy. Report of a joint task force of the European Federation of Neurological Societies and the Peripheral Nerve Society. Eur J Neurol. 2010;17(7):903-12, e44-9.

18. Lauria G, Bakkers M, Schmitz C, et al. Intraepidermal nerve fiber density at the distal leg: a worldwide normative reference study. J Peripher Nerv Syst. 2010;15(3):202-7. 
19. Lauria G, Dacci P, Lombardi R, et al. Side and time variability of intraepidermal nerve fiber density. Neurology. 2015;84(23):2368-71.

20. Nolano M, Biasiotta A, Lombardi R, et al. Epidermal innervation morphometry by immunofluorescence and bright-field microscopy. J Peripher Nerv Syst. 2015;20(4):387-91.

21. Tominaga M, Takamori K. Sensitization of itch signaling: itch sensitization-nerve growth factor, semaphorins. In: Carstens E, Akiyama T, editors. Itch: Mechanisms and Treatment. Boca Raton: CRC Press/Taylor \& Francis; 2014.

22. Haas S, Capellino S, Phan NQ, et al. Low density of sympathetic nerve fibers relative to substance P-positive nerve fibers in lesional skin of chronic pruritus and prurigo nodularis. J Dermatol Sci. 2010;58(3):193-7.

23. Rolke R, Baron R, Maier C, et al. Quantitative sensory testing in the German Research Network on Neuropathic Pain (DFNS): standardized protocol and reference values. Pain. 2006;123(3):231-43.

24. Backonja MM, Attal N, Baron R, et al. Value of quantitative sensory testing in neurological and pain disorders: NeuPSIG consensus. Pain. 2013;154(9):1807-19.

25. Hansson P, Backonja M, Bouhassira D. Usefulness and limitations of quantitative sensory testing: clinical and research application in neuropathic pain states. Pain. 2007;129(3):256-9.

26. Walk D, Sehgal N, Moeller-Bertram T, et al. Quantitative sensory testing and mapping: a review of nonautomated quantitative methods for examination of the patient with neuropathic pain. Clin J Pain. 2009;25(7):632-40.

27. Blankenburg $M$, Boekens $H$, Hechler $T$, et al. Reference values for quantitative sensory testing in children and adolescents: developmental and gender differences of somatosensory perception. Pain. 2010;149(1):76-88.

28. Eisen A. The use of somatosensory evoked potentials for the evaluation of the peripheral nervous system. Neurol Clin. 1988;6(4):825-38.
29. Mallik A, Weir AI. Nerve conduction studies: essentials and pitfalls in practice. J Neurol Neurosurg Psychiatry. 2005;76(2):ii23-31.

30. Cohen AD, Masalha R, Medvedovsky E, Vardy DA. Brachioradial pruritus: a symptom of neuropathy. J Am Acad Dermatol. 2003;48(6):825-8.

31. Cohen AD, Vander T, Medvendovsky E, et al. Neuropathic scrotal pruritus: anogenital pruritus is a symptom of lumbosacral radiculopathy. J Am Acad Dermatol. 2005;52(1):61-6.

32. Merkies IS, Faber CG, Lauria G. Advances in diagnostics and outcome measures in peripheral neuropathies. Neurosci Lett. 2015;596:3-13.

33. Papanas N, Ziegler D. Corneal confocal microscopy: a new technique for early detection of diabetic neuropathy. Curr Diab Rep. 2013;13(4):488-99.

34. Jalbert I, Stapleton F, Papas E, Sweeney DF, Coroneo M. In vivo confocal microscopy of the human cornea. Br J Ophthalmol. 2003;87(2):225-36.

35. Tavakoli M, Quattrini C, Abbott C, et al. Corneal confocal microscopy: a novel noninvasive test to diagnose and stratify the severity of human diabetic neuropathy. Diabetes Care. 2010;33(8):1792-7.

36. Malik RA, Kallinikos P, Abbott CA, et al. Corneal confocal microscopy: a non-invasive surrogate of nerve fibre damage and repair in diabetic patients. Diabetologia. 2003;46(5):683-8.

37. Asghar O, Petropoulos IN, Alam U, et al. Corneal confocal microscopy detects neuropathy in subjects with impaired glucose tolerance. Diabetes Care. 2014;37(9):2643-6.

38. Ständer S, Zeidler C, Augustin M, et al. Leitlinie: Diagnostisches und therapeutisches Vorgehen bei chronischem Pruritus (In preparation).

39. Weisshaar E, Szepietowski JC, Darsow U, et al. European guideline on chronic pruritus. Acta Derm Venereol. 2012;92(5):563-81.

40. Boyd K, Shea SM, Patterson JW. The role of capsaicin in dermatology. Prog Drug Res. 2014;68:293-306. 